\title{
Review
}

\section{Developing a Pan-Canadian Registry of Sudden Cardiac Arrest: Challenges and Opportunities}

\author{
Katherine Allan, PhD, ${ }^{a}$ Paul Dorian, MD, MSc, FRCPC, ${ }^{a, b}, *$ and

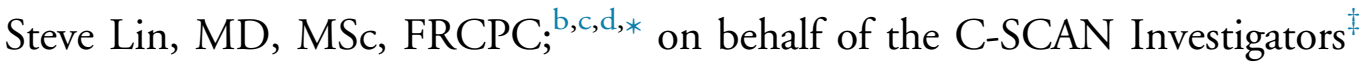 \\ ${ }^{a}$ Division of Cardiology, Department of Medicine, University of Toronto, Toronto, Ontario, Canada \\ ${ }^{b}$ Li Ka Shing Knowledge Institute, St Michael's Hospital, Toronto, Ontario, Canada \\ 'Division of Emergency Medicine, Department of Medicine, University of Toronto, Toronto, Ontario, Canada \\ ${ }^{d}$ Institute of Health Policy, Management and Evaluation, University of Toronto, Toronto Ontario, Canada
}

\begin{abstract}
Sudden cardiac arrest (SCA) has a devastating impact on both the family of the patient with SCA and his or her community. Because of various methodological differences between studies, reported incidence rates for SCA can vary widely, emphasizing the lack of clarity with respect to the true scope of this phenomenon. In recognition of the importance of accurately ascertaining the incidence and causes of SCA, there have been repeated calls for the development of national, prospective, population-based registries that use uniform definitions and multiple source methods to confirm cases. In this article, we discuss the challenges and opportunities in establishing a panCanadian registry and how its development will provide data on the current knowledge and treatment gaps to ultimately help reduce the burden of SCA in Canada.
\end{abstract}

Sudden cardiac arrest (SCA) is generally defined as an abrupt cardiovascular collapse in a seemingly healthy person, with instantaneous loss of vital signs or $\leq 1$ hour within the onset of symptoms. ${ }^{1}$ If resuscitative efforts succeed, these events are termed "SCAs." If they do not, these are termed "sudden cardiac deaths" (SCDs). Depending on the definition used, how cases are ascertained and what information is obtained to determine the underlying cause of death, the incidence rates and distributions of SCA and SCD etiologies differ substantially between studies. ${ }^{2,3}$ This makes comparisons of SCA and

Received for publication December 4, 2018. Accepted January 28, 2019.

Ethics Statement: The study was approved by the St. Michael's Hospital Research Ethics Board.

*These authors contributed equally to this work.

${ }^{\ddagger}$ For a complete listing of the C-SCAN Investigators, please see Appendix 1 at the end of this article.

Corresponding author: Dr Katherine Allan, St Michael's Hospital, 193 Yonge Street, Suite 3-007, Toronto, Ontario M5B 1M8, Canada. Tel.: +1416-864-6060 ×76347; fax: +1-416-864-5849.

E-mail: allank@smh.ca

See page 60 for disclosure information.

\section{RÉSUMÉ}

L'arrêt cardiaque soudain (ACS) a des conséquences dévastatrices sur la famille et la collectivité du patient ayant subi un ACS. En raison des diverses différences méthodologiques entre les études, les taux d'incidence de cas déclarés d'ACS peuvent grandement varier, d'où le manque de clarté quant à la véritable ampleur de ce phénomène. Vu l'importance de déterminer avec précision l'incidence et les causes de l'ACS, on a demandé à maintes reprises l'élaboration de registres prospectifs nationaux de population qui recourent à des définitions uniformes et des méthodes de sources multiples pour confirmer les cas. Dans le présent article, nous nous penchons sur les enjeux et les possibilités d'établissement d'un registre pancanadien et sur la manière avec laquelle son élaboration fournira des données sur les connaissances actuelles et les lacunes en matière de traitement pour finalement contribuer à la réduction du fardeau de l'ACS au Canada.

SCD rates between studies challenging and serves to highlight the lack of clarity with regard to the true scope. ${ }^{4}$

In recognition of the importance of accurately ascertaining the incidence and causes of SCA and SCD, there have been repeated calls for the development of national, prospective, population-based registries that use uniform definitions and multiple source methods to confirm cases. ${ }^{3-5}$

There are 3 main actionable steps that we will describe in further detail: (1) establish a national registry of all SCA and SCD events that uses multiple source methods and comprehensive data-collection processes to ensure complete case ascertainment; (2) develop pragmatic, uniform definitions for SCA and SCD that should not be used interchangeably; and (3) accurately determine the underlying etiologies (both cardiac and noncardiac) of all cases.

\section{Setting up a National Registry}

In 2016, the Canadian Resuscitation Outcomes Consortium (CanROC) was formed to prospectively collect all out-of-hospital cardiac arrests (OHCAs) and traumas that 
occur in participating emergency medical services (EMS) agencies, with the goal of including as many communities throughout Canada as possible. CanROC currently involves all 10 provinces, covering a population of approximately 30 million people. The data from this registry will be used to monitor and improve resuscitation treatment efforts and to provide the infrastructure to implement clinical trials. Although this prospective registry tracks OHCAs nationally, it captures all types of OHCAs, both cardiac and noncardiac, and thus may not provide accurate estimates of "true" SCA and SCD incidence. We define true SCA as an abrupt collapse with documented loss of vital signs and attended by EMS in response to a 911 call. The etiology is considered to be only cardiac after the exclusion of SCAs due to noncardiac causes. Cases can be witnessed or unwitnessed, with no time limit restrictions from the onset of the event to when the individual is found. SCA includes both patients who do not survive (previously defined as SCD) and those successfully resuscitated $^{6}$ (Table 1).

In 2017, the Canadian Sudden Cardiac Arrest Network (C-SCAN), funded by the Cardiac Arrhythmia Network of Canada (CANet) as part of the Networks of Centres of Excellence (NCE) was formed. The overall objective of this network is to develop a comprehensive national registry that accurately measures the burden of SCA and SCD: its incidence, etiologies, associated healthcare consumption, and patient outcomes. This registry will allow a better understanding of the circumstances, specific pathophysiological etiologies, and healthcare consumption before and after SCA or SCD events.

C-SCAN will build upon the existing CanROC infrastructure and expand their dataset to include multisource records: prehospital data, coroner/autopsy data, administrative datasets, and survivor interviews. The value of this registry lies with the integration of numerous differing datasets that will provide a comprehensive understanding of why and when SCA and SCD events occur and to whom. There are several key challenges affecting the accurate identification of cases that the C-SCAN registry will need to overcome: (1) developing consistent definitions for how SCA and SCD are defined; and (2) developing consistent and accurate methods to determine and classify the cause of each event.

\section{Differing Definitions}

The estimations of SCA and SCD incidences and causes are complicated by a lack of consistent definitions. The terms "out-of-hospital cardiac arrest, "sudden death," "sudden cardiac arrest," and "sudden cardiac death" are often used interchangeably, despite having very different definitions. ${ }^{4}$ Some studies include time constraints, such as within 1 to 6 hours of the onset of acute symptoms or a death occurring within 24 hours of symptom onset. ${ }^{7-9}$ Others define SCD as death due to ischemic or coronary heart disease ${ }^{8,10}$ or broaden their criteria to include all cardiovascular or cardiac etiologies. $^{7,9,11}$

Although it is implied that "cardiac arrest" is caused by cardiac disease in most or all SCA cases, recent studies have highlighted that many cases of cardiac arrest are due to noncardiac disease, such as metabolic diseases, unrecognized overdoses or drug toxicity, and vascular noncardiac conditions. ${ }^{6,7,12,13}$ The lack of consensus on how to define both SCA and SCD remains a significant barrier to the accurate reporting of this condition.

Using a time-based system to classify deaths is problematic for several reasons. First, it can result in different SCD incidence rates depending on the time definition for what constitutes "sudden." The Postmortem Systematic Investigation of Sudden Cardiac Death (POST SCD) study prospectively identified all SCDs that occurred in San Francisco County from 2011 to 2014 using the World Health Organization time-based definitions for SCD (within 1 hour if witnessed and within 24 hours if unwitnessed) and compared them with autopsy-defined SCD. By using the World Health Organization conventional definitions, only $55.8 \%$ of SCDs were proven to be due to cardiac causes after autopsy, with the remaining cases due to nonarrhythmic and noncardiac causes. $^{13}$

Second, the time frame from symptom onset to the SCD event is difficult to determine for unwitnessed events, leading to misclassification bias. Some in-hospital deaths may occur hours to days after the initial collapse as a late result of the SCA event. Some "sudden" deaths may occur in-hospital and are generally not unexpected. The most important question in understanding and defining SCA and SCD does not involve the timing of the moment of death, but instead should focus on "what underlying disease, disorder or event caused the sudden death?"

\section{C-SCAN Standardized Definitions}

There is clearly an urgent need to develop pragmatic, uniform definitions for SCA and SCD that are based on all of the clinical information available and can be agreed upon and adopted by the major stakeholders in the medical and scientific community. These should be 2 distinct definitions that

Table 1. Sudden cardiac arrest and sudden cardiac death definitions

\begin{tabular}{ll}
\hline \hline AHA definitions $^{14}$ & SCA \\
restore circulation. & SCA results from many disease processes. \\
Our definitions & SCA is defined as an abrupt collapse with documented loss of vital \\
signs and attended by EMS in response to a 911 call. $*$ & SCD is when attempts to restore circulation are \\
The etiology is considered to be only cardiac after the exclusion of \\
SCAs due to vascular noncardiac disease, acute noncardiac illnesses, \\
drug overdose, metabolic causes, or terminal disease.
\end{tabular}

\footnotetext{
AHA, American Heart Association; EMS, emergency medical services; SCA, sudden cardiac arrest; SCD, sudden cardiac death.

* Cases could be witnessed or unwitnessed, with no time-limit restrictions from the onset of the event to when the individual was found. SCA includes both patients who do not survive and those successfully resuscitated.
} 


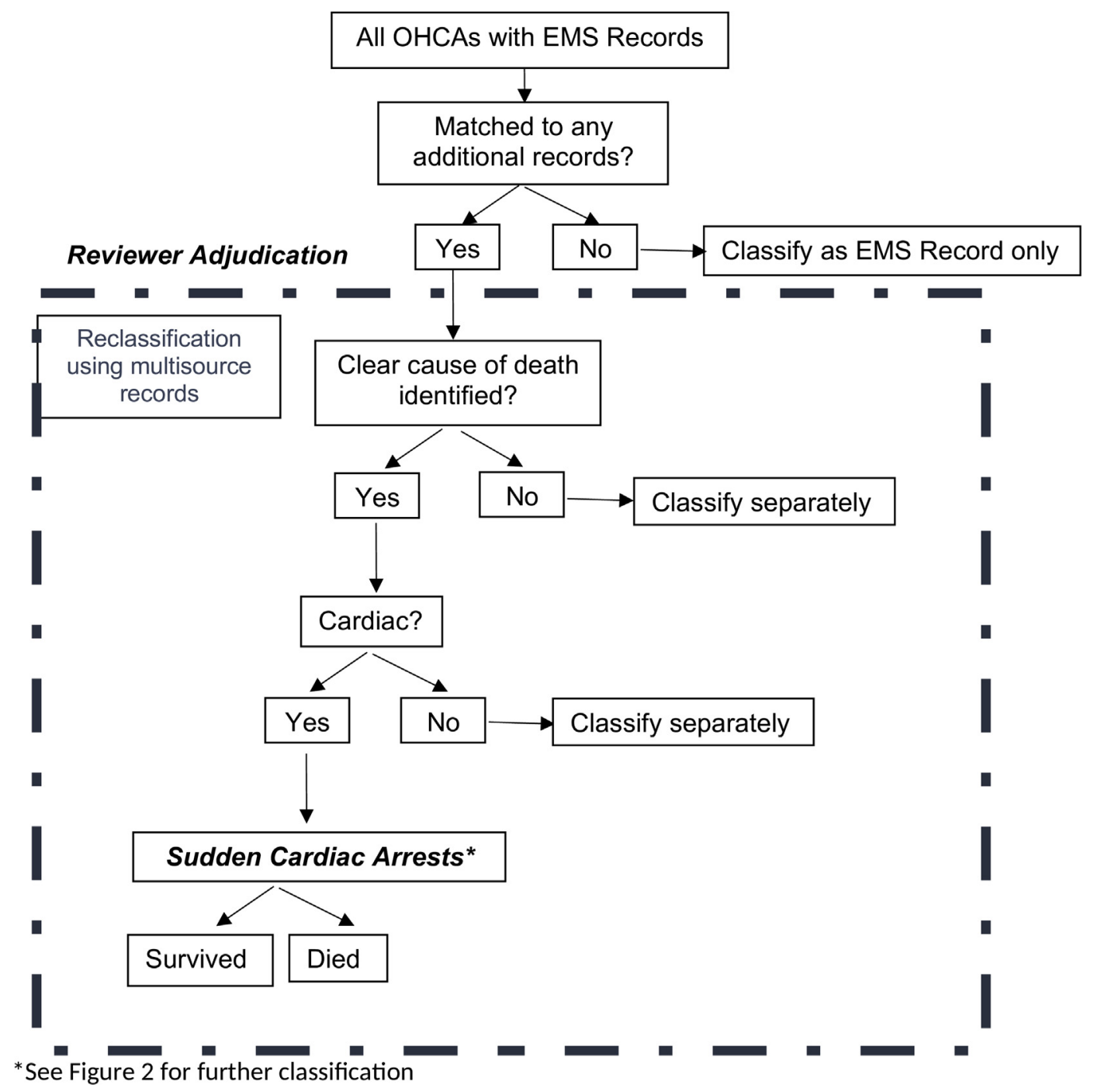

Figure 1. Review and classification of etiologies using multisource documentation. EMS, emergency medical services; OHCA, out-of-hospital cardiac arrest.

should not be used interchangeably, which will facilitate a more reliable assessment of the incidences of these phenomena.

We have expanded the definitions put forth by the American Heart Association, ${ }^{14}$ which avoid the use of time to define an event as "sudden" (Table 1). Our definitions use an adjudication process to determine the underlying etiology, both cardiac and noncardiac, which will facilitate a more accurate assessment of the incidence rates and enhance strategies for risk stratification and prevention of SCA and SCD (Fig. 1).

The review process uses the following steps: Initially, all deceased patients with OHCAs are matched to a coroner investigative statement and autopsy/toxicology report where available; all resuscitated patients with OHCAs are matched to their inhospital reports, where available (Fig. 1). Patients with OHCA who remain unmatched across source documents or have unclear etiology (either cardiac or noncardiac) are coded separately.

The remaining OHCAs with known etiologies are further subcategorized using a joint deliberation process between experts, using all available sources of information to assign the cause, cardiac or noncardiac. OHCAs with contributing toxicology results are further reviewed with a coroner, cardiac pathologist, and toxicologist. For all cases, a decision is reached by consensus.

OHCAs that are attributed to noncardiac causes such as drug overdose, suicide, homicide, drowning, motor vehicle collisions, blunt, penetrating, or burn injury trauma, cancer, complex chronic care, acute noncardiac illnesses, and vascular noncardiac causes are coded separately (Fig. 2). The adjudicated OHCAs of confirmed cardiac cause comprise the SCA cohort of interest.

\section{Case Ascertainment Methods}

Case ascertainment methods for identifying SCAs and SCDs vary widely, from using death certificates and administrative data, to autopsy records, or direct ascertainment from EMS records of paramedics attending cardiac arrests. 


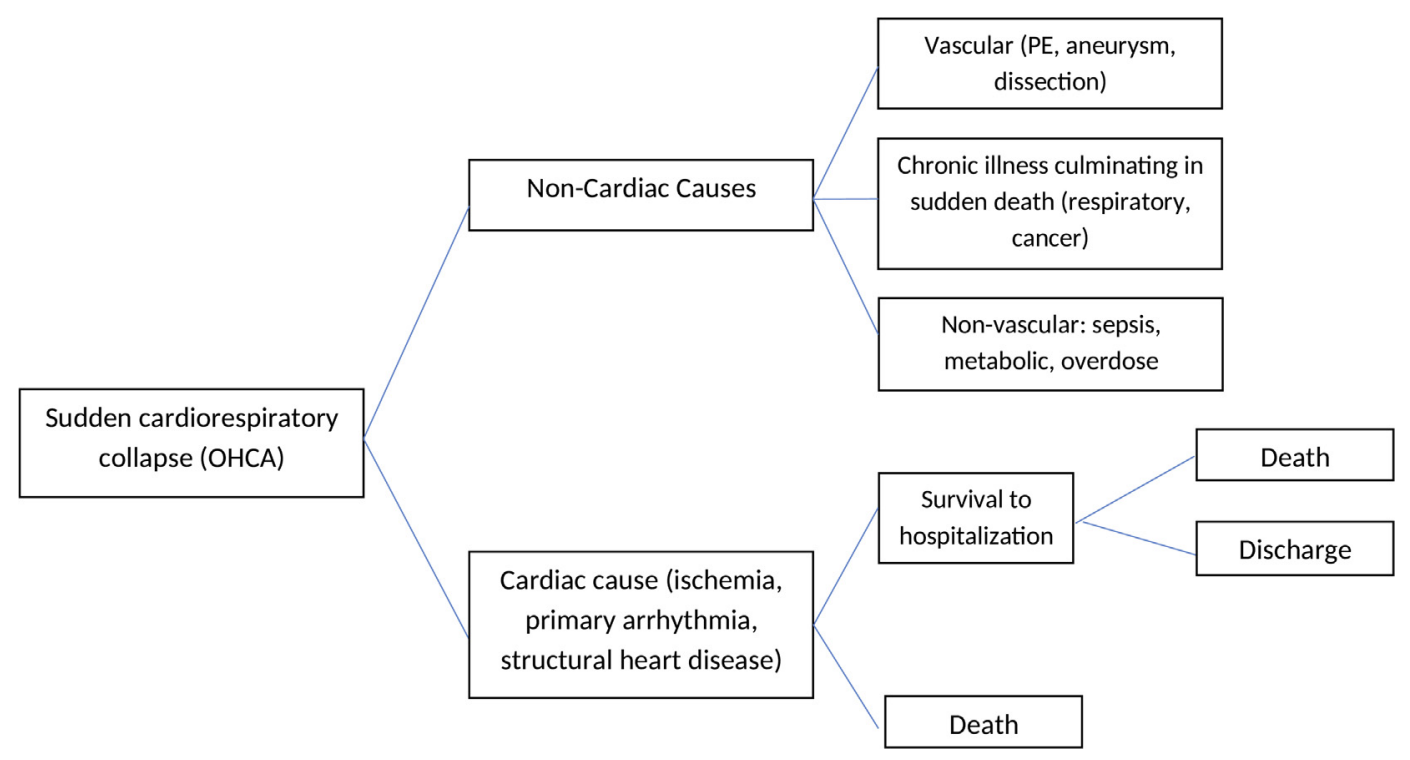

Figure 2. Classification of cardiac and noncardiac etiologies. OHCA, out-of-hospital cardiac arrest; PE, pulmonary embolism.

\section{Death certificates and administrative data}

The most commonly used method to ascertain SCD cases involves using death certificates and administratively collected International Classification of Diseases $10^{\text {th }}$ Revision codes, despite their tendency to be prone to inconsistent or erroneous classification. ${ }^{3}$ One of the main reasons for the lack of agreement between death certificates and actual cause of death on autopsy is that deaths are often inappropriately labeled as cardiac in administratively classified datasets. Tavora et al. ${ }^{15}$ studied a series of consecutive autopsies in cases considered to be sudden and out-of-hospital to determine the accuracy of death certificates compared with cause of death determined by autopsy. The overall discrepancy rate was $52 \%$ and lowest in patients with known symptomatic heart disease and highest in patients without a medical history. In more than threequarters of cases, coronary disease was listed on the death certificate when autopsy demonstrated a different cause of death.

\section{Autopsy registries}

Another method is to use only autopsied cases of SCD to determine incidence rates. A recent Swedish study that used the Swedish National Board of Forensic Medicine database and the Swedish Cause of Death Registry to identify patients with SCD ages 1 to 35 years found an incidence rate of 1.3 per 100,000 person-years in 1 - to 35 -year-olds. ${ }^{16}$ Using only autopsied cases will improve the specificity of identifying cardiac cases; however, not all SCD cases are autopsied. Additionally, some patients with SCA survive after resuscitation, and a comprehensive accounting of SCA and SCD cases should include those who survive to hospital discharge.

\section{EMS registries}

There are several large prospective OHCA registries based in North America (Resuscitation Outcomes Consortium, Cardiac Arrest Registry to Enhance Survival, Canadian Resuscitation Outcomes Consortium), ${ }^{17,18}$ Australia and New
Zealand (Australian Resuscitation Outcomes Consortium), ${ }^{19}$ and Asia (Pan-Asian Resuscitation Outcomes Study). ${ }^{20}$ All of these registries collect OHCAs prospectively in large, geographic areas with the aim of monitoring and reporting on prehospital resuscitation outcomes and practices, as well as providing the infrastructure needed to implement large clinical trials. The reported OHCA incidence rates vary widely between registries, ranging from 57 to 110 per 100,000 population, depending on whether the OHCAs include (1) EMS treated, (2) "EMS-attended, not treated," (3) "presumed cardiac" etiology, or (4) all types.

The challenge with using OHCA as a surrogate for SCA is that the reliability of paramedics determining etiology during resuscitation in the out-of-hospital setting is limited by the lack of detailed historical and clinical information. In patients aged 2 to 45 years in a large urban area, only $29.9 \%$ of a total of 1993 OHCAs that paramedics designated "no obvious cause" were confirmed to be of cardiac causes; the rest were due to other noncardiac etiologies: drug overdose $(31.3 \%)$, trauma $(5.4 \%)$, cancer $(3.5 \%)$, complex chronic care $(3.3 \%)$, and noncardiac acute illness (eg, vascular, infectious, and metabolic) (18.9\%). ${ }^{12}$ This likely results in an overestimation of SCA and SCD incidences.

\section{Multisource surveillance and data-collection methods}

The Oregon Sudden Unexpected Death Study (SUDS) is one of a select few to use prospectively collected multisource methods to assess SCD rates in a large U.S. community. ${ }^{7,21}$ Patients who suffer a sudden unexpected death are identified from several sources: the EMS response system, the county Medical Examiner's (ME's) office, and 16 area hospitals. By using all available medical records, a comprehensive evaluation is then performed for each case that includes analyzing the circumstances, medical records, and autopsy data. All abstracted information is assembled in a database, after which an adjudication process is used to determine which cases meet their criteria for SCD. Despite these 
comprehensive methods, autopsy rates remain low, thus limiting the accuracy of the findings.

In 2013, the National Institutes of Health and the Centre for Disease Control collaborated to fund the Sudden Death in the Young Registry. ${ }^{22}$ This registry collects comprehensive, population-based information on sudden unexpected death in youths up to age 20 years in the United States and includes participation from 14 differing states. Participation is voluntary, with interested institutions having to "opt in." Although this is a great first step toward the development of a national-level registry, it only collects data on SCD in the young.

\section{C-SCAN multisource datasets}

There are many etiologies of SCA, and treatments may widely differ, so it is important to accurately identify and classify all SCAs. For example, SCAs can be heritable, and up to $50 \%$ of first-degree relatives could be affected. To treat and prevent SCA at both a population and individual level, the specific causes and their distribution need to be identified. Our methodology uses a comprehensive evaluation of each case by reviewing all available medical records (as detailed next) to determine the circumstances and precise cause of death, cardiac or noncardiac (Fig. 3). This facilitates as accurate an estimation as possible of the incidence, circumstances, and causes of SCA and SCD.

\section{EMS data}

Paramedics and firefighters fill out ambulance call and fire reports, respectively, to document any emergency situation they attend, including cardiac arrests. These reports contain patient identifiers, medical history, medications, narrative details about the circumstances of the event, call characteristics, prehospital interventions including the defibrillator monitor recording during the resuscitation, and outcomes in the field. Collectively, the resuscitation variables on these reports are referred to as the "Utstein elements," have standardized definitions, and are used to track and report resuscitative efforts by EMS systems worldwide. ${ }^{23}$ These data provide an in-depth picture of the events leading up to the SCA and the quality of the resuscitative efforts.

\section{Coroner data}

Each province and territory is responsible for its own death investigations; as such, each has developed their own system and legislation to investigate deaths that are due to injury or drugs, or are unexpected or unexplained. ${ }^{24}$ Canada has 2 differing types of death investigation systems: the Medical Examiner (ME) system and the Coroner's system. Most provinces and territories use the Coroner's system (Ontario, British Columbia, Saskatchewan, New Brunswick, Quebec, North West Territories, Nunavut, Yukon, and Prince Edward Island), whereas Alberta, Manitoba, Nova Scotia, and

Cases are linked to administrative datasets. PHI removed and unique case identifier assigned. De-identified linked individual level data is sent back to CSCAN Registry

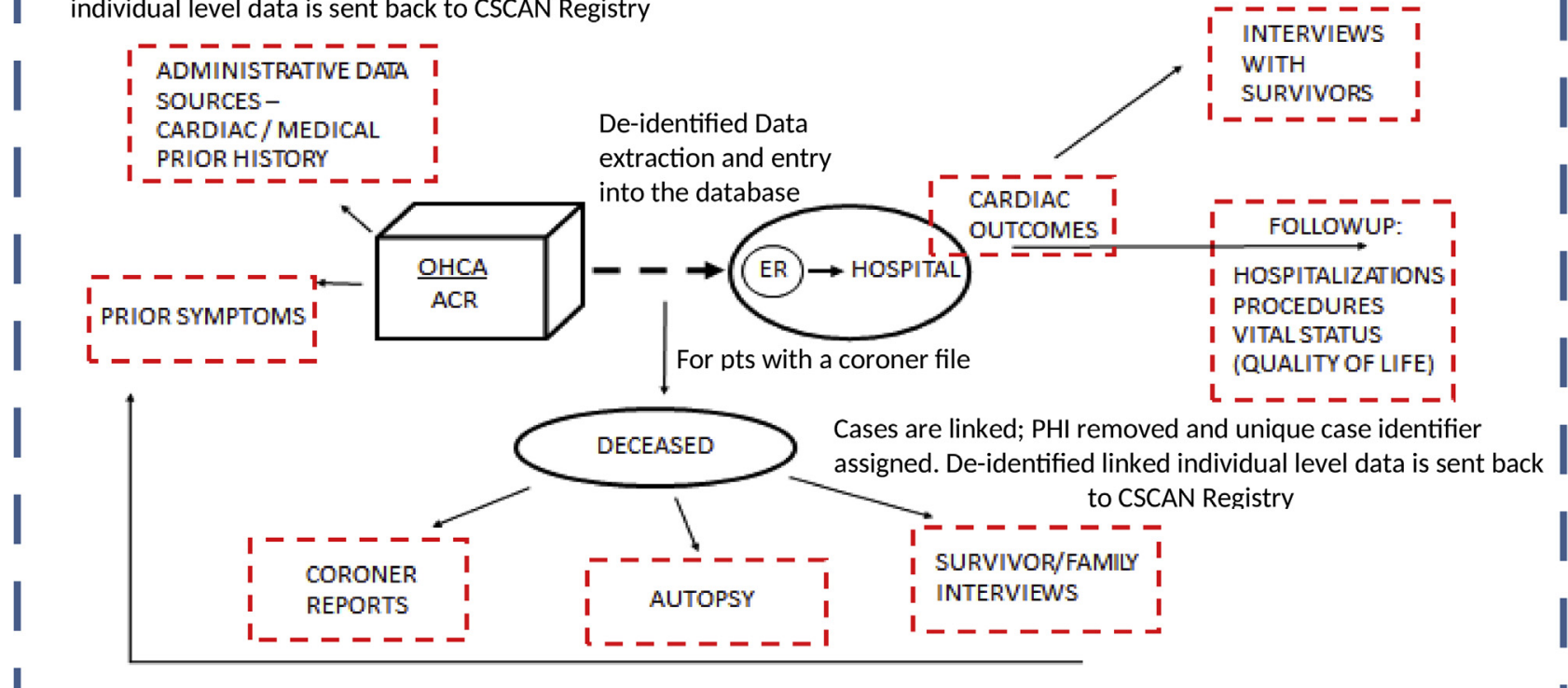

OHCA: Out-of-hospital cardiac arrest

PHI: personal health identifiers ACR: Ambulance call report

\section{SECURE DATA ENVIRONMENT}

Figure 3. Proposed Canadian Sudden Cardiac Arrest Network (C-SCAN) Registry. Schema of data sources. Currently collected data outlined in black; proposed additional data outlined in hatched red. ACR, ambulance call report; ER, emergency room; OHCA, out-of-hospital cardiac arrest; PHI, personal health identifier. 
Newfoundland and Labrador use the ME system. MEs are physicians, whereas coroners can be lay people or physicians. Regardless of the system, they both have the same objective, which is to determine the cause and circumstances surrounding deaths and the identification of the deceased.

Each province and territory holds the investigated death data in a centralized office. The number of deaths investigated by a $\mathrm{ME}$ or coroner can vary significantly from as low as $7 \%$ to as high as $45 \%$ annually. ${ }^{24}$

To allow provinces and territories to collect and aggregate their data, a common dataset was developed. ${ }^{24}$ This standardized dataset includes demographics on the deceased, how the deceased died, including circumstances and activity at the time of death, and the medical cause of death with contributory factors. The dataset also includes a summary of the medical history acquired from medical records or interviews with those who knew the deceased (ie, bystanders, family members, or friends). When relevant, the report may state if an inquest or public inquiry should be held, as well as any recommendations that should be made to prevent similar type deaths from reoccurring. This is a unique data source that provides insight into the entire SCA event, from relevant medical history and medications to the circumstances leading up to the event including premonitory symptoms and potential triggers.

\section{Autopsy data}

When the cause or manner of death is not immediately obvious, coroners and MEs will order an autopsy. Autopsies that are performed at a forensic centre will follow a standardized protocol in which all organs are examined both macroscopically and microscopically and be conducted by a forensic pathologist. When the cause of death is deemed to be due to an underlying cardiac disease, the heart will be sent to a specialized cardiovascular pathologist for more detailed examination. Rates of autopsies vary across the country and have been declining since 2013 , with an overall rate of $5.9 \%$ of all reported deaths. ${ }^{25}$ Overestimation of SCD incidence is likely to occur without detailed history and autopsy data through misclassification of cases that are not cardiac.

\section{Toxicology}

Toxicology is performed depending on the circumstances at the scene and findings from the coroner/ME's investigation and the postmortem examination. It is usually performed to aid in the death investigation, such as in circumstances where the manner or cause of death is unclear or if the cause of death is thought to be related to drugs. If toxicology is thought to play a contributing role in the death, it will be performed regardless if an anatomic cause of death is found at autopsy. Toxicology testing follows standardized protocols that include screening for ethanol, drugs of abuse, and 300 over-thecounter drugs. ${ }^{26}$ Testing occurs until a drug is detected at a toxicologically significant or fatal level, after which further testing is not performed. Toxicology results are vital to determine if the SCD is due to a nonobvious drug overdose or if drugs play a contributory factor to the SCD event.

\section{Administrative datasets}

Across most of Canada with the exception of Quebec, the National Ambulatory Care Reporting System (NACRS) database captures all visits to the emergency department, and the Discharge Abstract Database (DAD) obtains all hospitalizations and in-hospital procedures. The NACRS contains emergency department visits and ambulatory care visits that are provided by hospitals and community clinics (ie, day surgeries and outpatient procedures). Data are inputted by participating facilities at the time of service and then sent to the Canadian Institute for Health Information (CIHI) for central storage. Excluding Quebec, most provinces and territories submit NACRS data to CIHI.

The DAD database collects hospital admissions and discharges, including clinical and demographic information on all patients (died or transferred), as well as administrative data. The DAD is also used by some provinces and territories to collect day surgeries. Acute care facilities, regional health authorities, or provincial ministries of health send their DAD data directly to CIHI. Most provinces and territories mandate the reporting of this data with the exclusion of Quebec.

From these databases, we can access cardiac risk factors, major comorbidities, cardiac procedures, and hospitalizations both before and after the index event. This will provide us with an understanding of each SCA victim's medical history and postresuscitation care, including subsequent admissions after the index event.

\section{Interviews with survivors}

We have developed and piloted a semiquantitative questionnaire that captures both the medical history in further detail, including family history of SCA/SCD, and the circumstances and triggers for the event (Supplemental Appendix S1). This level of detail would not otherwise be captured by the mentioned datasets and could provide insight into the events leading up to the SCA or SCD event.

\section{Expected Challenges}

There are numerous challenges to setting up a national registry; to link multiple datasets, personal identifiers are needed to accurately link each patient with SCA to his or her medical information. Privacy regulations and the need for research ethics board's approvals add complexity, because not all provincial legislation allows the transmission of personal identifiers across provincial boundaries. Data security is also a major concern given the need to store all of these linked data and keeping them secure. Moreover, to set up this type of registry involves collaboration and cooperation from numerous differing institutions and individuals who otherwise would not normally interact, from EMS to coroners, cardiologists and internists, epidemiologists, and scientists. Finally, there is the issue of sustainability; how do we maintain funding and interest in keeping the registry going?

\section{Privacy and Ethics}

The objective of improving the health of Canadians requires ongoing research. One of the major challenges is access to highquality data, which includes patient data on their encounters with the healthcare system in addition to social data on issues that affect health outcomes. The healthcare and research communities view these data as a critical resource with great potential to affect and improve health outcomes; yet this must 
be balanced with an individual's right to privacy and for their personal health-related data to be kept confidential.

In Canada, there are federal and provincial laws that establish standards for health information governance and privacy rights. The Office of the Privacy Commissioner of Canada enforces 2 federal privacy laws: the Privacy Act and the Personal Information Protection and Electronic Documents Act. The Federal Privacy Act encompasses how the federal government handles personal information, whereas the Personal Information Protection and Electronic Documents Act applies to both federal and provincial entities using personal and health data. ${ }^{27,28}$ Several provinces have developed specific legislation to further define and govern the use and privacy of health data. As an example, Ontario has the Personal Health Information Protection Act (PHIPA) that applies to all health information custodians within the province and lays out rules for the collection, use, and disclosure of personal health information.

The overarching challenge to perform Canadian-wide research, including ours, is to meet the 2 fundamental goals of accessing health and health-related data for research to improve health outcomes and to respect the privacy and confidentiality of Canadians when using their personal health information for research. Ideally, there would be an organization(s) with established governance principles and efficient operating procedures to support timely access to healthcare data nationally and across provinces for research. However, this is currently not the case. Data access for research often requires the careful navigation through a complex environment of heterogeneous entities that include numerous data custodians, research ethics boards, and privacy offices, whose collective governance and practices fall short of constituting a well-defined and coherent system. ${ }^{29}$ Fortunately, the Canadian Institutes of Health Research (CIHR) has recently funded the development of a national data platform proposed by the Pan-Canadian RealWorld Health Data Network (PRHDN) (https://www.prhdn. $\mathrm{ca} /$ SitePages/Home.aspx). Its objectives include harmonizing definitions and data variables, creating a data access support system to help navigate multi-jurisdictional data requests and expand data sources and linkages.

Without the proposed platform by PRHDN, we will work with individual provinces to build a local database within each respective province to collect, abstract, and store SCA health data. Personal health information will be collected and used for data linkage to available data sources within each respective province. Deidentified and anonymous data will be used to produce aggregate reports for the data coordinating centre.

\section{Data Security}

To link all of the varying data sources in each localized C-SCAN database necessitates the collection of personal identifiers, thus requiring appropriate data security measures.

To prevent privacy breaches, all identifiers will be entered into a password-protected encrypted master linking $\log$ and will be stored locally on secure site-specific servers. Only designated study staff will have access to these master linking logs, and the logs will be stored in a separate location from the main database to prevent any identification of subjects.
Research ethics board-approved standards and practices for study data collection, storage, and retrieval will be used at each site to minimize any loss of confidentiality and privacy. All study personnel involved in data collection and analysis will be required to sign a confidentiality agreement and will receive appropriate training.

\section{Organizational Challenges}

To collect and integrate all of these varying data sources requires collaboration among many different institutions and specialists within each province and across Canada. The initial collaboration between CanROC and CANet has unified EMS and emergency physicians with cardiologists, who until 2017 often worked in isolation with the same end goal. However, this has required finding a common language of terminology and methodology that can be understood and implemented by both parties.

Because SCA is such a complex condition, it requires collaboration between more than just EMS and cardiology researchers and practitioners to both understand and treat. We have expanded our group to include pediatric experts, internists, nurses, coroners, MEs, social scientists, epidemiologists, patient advocates, and patient group representatives.

This network of specialists across the country provides opportunities for new collaborations where previously there were none and a chance for trainees to get involved locally and nationally by seeking out new mentorship opportunities.

\section{Sustainability}

The development and implementation of C-SCAN will take 3 years and cost more than $\$ 1.2$ million. The study is currently funded by CANet as part of the NCE. If C-SCAN is to survive, alternative sources of funding outside of the traditional research grants will be required. Ideally, C-SCAN could find a home in the proposed PRHDN national data platform, because this would significantly reduce the complex privacy and data security challenges as described earlier.

Another solution could encompass moving the linkage and storage of data to a prescribed entity; in Ontario, there are 4 prescribed entities: Cancer Care Ontario, Pediatric Oncology Group of Ontario, Institute for Clinical Evaluative Sciences, and CIHI, which is national in scope but excludes Quebec. The advantage of this strategy is that under the Personal Health Information Protection Act, prescribed entities are allowed to hold personal health information for the purposes of facilitating or improving the provision of healthcare without patient consent. Because most cardiac arrest research requires a waiver of consent because of the inability of the patient to consent at the time of enrollment (ie, during cardiac arrest), this strategy would be ideal, because it would balance the issues of patient privacy, confidentiality, and data security with the need to improve healthcare outcomes. Moreover, in certain circumstances, prescribed entities are allowed to use and disclose personal health information for research purposes, provided there is a research plan approved by a research ethics board. This means that with appropriate research ethics approvals, the prescribed entity could disclose deidentified linked data to our institution for analysis purposes. 


\section{Conclusions}

If you cannot measure it, you cannot improve it. This registry will allow us to better understand the circumstances, specific pathophysiological etiologies, and healthcare consumption before and after SCA and SCD events. Moreover, it will deliver data on current knowledge and treatment gaps, and provide the necessary infrastructure to evaluate the effectiveness of targeted interventions, to ultimately help reduce the burden of SCD in Canada.

\section{Funding Sources}

C-SCAN is funded by a CANet as part of the NCE program grant, as part of the Networks of Centres of Excellence (NCE).

\section{Disclosures}

Dr Dorian is a Network Investigator of the CANet as part of the NCE and holds a CANet Program grant. Dr Lin is a Network Investigator of CANet as part of the Networks of Centres of Excellence, holds a CANet Project grant, is supported by a Heart and Stroke Foundation of Canada Ontario Clinician-Scientist Award, is an Evidence Reviewer for the International Liaison Committee on Resuscitation, and was a chapter author for the 2015 American Heart Association Guidelines Update on Cardiopulmonary Resuscitation and Emergency Cardiovascular Care. Dr Allan is Chair of the CANet Training and Education Committee and receives salary support from a CANet Program grant.

\section{References}

1. Gajewski KK, Saul JP. Sudden cardiac death in children and adolescents (excluding sudden infant death syndrome). Ann Pediatr Cardiol 2010;3: 107-12.

2. Fishbein MC. Cardiac disease and risk of sudden death in the young: the burden of the phenomenon. Cardiovasc Pathol 2010;19:326-8.

3. Kaltman JR, Thompson PD, Lantos J, et al. Screening for sudden cardiac death in the young: report from a National Heart, Lung, and Blood Institute working group. Circulation 2011;123:1911-8.

4. Kong MH, Fonarow GC, Peterson ED, et al. Systematic review of the incidence of sudden cardiac death in the United States. J Am Coll Cardiol 2011;57:794-801.

5. Zipes DP, Camm AJ, Borggrefe M, et al. ACC/AHA/ESC 2006 guidelines for management of patients with ventricular arrhythmias and the prevention of sudden cardiac death-executive summary: A report of the American College of Cardiology/American Heart Association Task Force and the European Society of Cardiology Committee for Practice Guidelines (Writing Committee to Develop Guidelines for Management of Patients with Ventricular Arrhythmias and the Prevention of Sudden Cardiac Death) Developed in collaboration with the European Heart Rhythm Association and the Heart Rhythm Society. Eur Heart J 2006;27:2099-140

6. Allan KS, Morrison LJ, Pinter A, Tu JV, Dorian P. Unexpected high prevalence of cardiovascular disease risk factors and psychiatric disease among young victims of sudden cardiac arrest. J Am Heart Assoc Jan 19 2019 [Epub ahead of print].

7. Chugh SS, Jui J, Gunson K, et al. Current burden of sudden cardiac death: multiple source surveillance versus retrospective death certificate-based review in a large U.S. community. J Am Coll Cardiol 2004;44:1268-75.

8. Escobedo LG, Zack MM. Comparison of sudden and nonsudden coronary deaths in the United States. Circulation 1996;93:2033-6.

9. Zheng ZJ, Croft JB, Giles WH, Mensah GA. Sudden cardiac death in the United States, 1989 to 1998. Circulation 2001;104:2158-63.

10. Gillum RF. Sudden coronary death in the United States: 1980-1985. Circulation 1989;79:756-65.

11. Cobb LA, Fahrenbruch CE, Olsufka M, Copass MK. Changing incidence of out-of-hospital ventricular fibrillation, 1980-2000. JAMA 2002;288:3008-13.

12. Allan KS, Morrison LJ, Pinter A, Tu JV, Dorian P. "Presumed cardiac" arrest in children and young adults: a misnomer? Resuscitation 2017;117: 73-9.

13. Tseng ZH, Olgin JE, Vittinghoff E, et al. Prospective countywide surveillance and autopsy characterization of sudden cardiac death: POST SCD Study. Circulation 2018;137:2689-700.

14. Benjamin EJ, Virani SS, Callaway CW, et al. Heart Disease and Stroke Statistics-2018 Update: a report from the American Heart Association. Circulation 2018;137:e67-492.

15. Tavora F, Crowder CD, Sun CC, Burke AP. Discrepancies between clinical and autopsy diagnoses: a comparison of university, community, and private autopsy practices. Am J Clin Pathol 2008;129:102-9.

16. Wisten A, Krantz P, Stattin EL. Sudden cardiac death among the young in Sweden from 2000 to 2010: an autopsy-based study. Europace 2017;19:1327-34.

17. McNally B, Stokes A, Crouch A, Kellermann AL. CARES: Cardiac Arrest Registry to Enhance Survival. Ann Emerg Med 2009;54:674-83.

18. Morrison LJ, Nichol G, Rea TD, et al. Rationale, development and implementation of the Resuscitation Outcomes Consortium EpistryCardiac Arrest. Resuscitation 2008;78:161-9.

19. Beck B, Bray J, Smith K, et al. Establishing the Aus-ROC Australian and New Zealand out-of-hospital cardiac arrest Epistry. BMJ Open 2016;6: e011027.

20. Ong ME, Shin SD, Tanaka H, et al. Rationale, methodology, and implementation of a dispatcher-assisted cardiopulmonary resuscitation trial in the Asia-Pacific (Pan-Asian Resuscitation Outcomes Study Phase 2). Prehosp Emerg Care 2015;19:87-95.

21. Jayaraman R, Reinier K, Nair S, et al. Risk factors of sudden cardiac death in the young: multiple-year community-wide assessment. Circulation 2018;137:1561-70.

22. Burns KM, Bienemann L, Camperlengo L, et al. The sudden death in the Young Case Registry: collaborating to understand and reduce mortality. Pediatrics 2017;139(3).

23. Perkins GD, Jacobs IG, Nadkarni VM, et al. Cardiac arrest and cardiopulmonary resuscitation outcome reports: update of the Utstein Resuscitation Registry Templates for Out-of-Hospital Cardiac Arrest: a statement for healthcare professionals from a task force of the International Liaison Committee on Resuscitation (American Heart Association, European Resuscitation Council, Australian and New Zealand Council on Resuscitation, Heart and Stroke Foundation of Canada, InterAmerican Heart Foundation, Resuscitation Council of Southern Africa, Resuscitation Council of Asia); and the American Heart Association Emergency Cardiovascular Care Committee and the Council on Cardiopulmonary, Critical Care, Perioperative and Resuscitation. Circulation 2015;132:1286-300. 
24. Statistics Canada. Canadian Coroner and Medical Examiner Database: Annual Report. Available at: https://www150.statcan.gc.ca/n1/pub/82214-x/2012001/int-eng.htm. Accessed November 16, 2018.

25. Statistics Canada. Deaths subject to autopsy. Available at: https:// www150.statcan.gc.ca/t1/tbl1/en/tv.action?pid=1310071601. Accessed November 16, 2018

26. Woodall KL, Chow BL, Lauwers A, Cass D. Toxicological findings in fatal motor vehicle collisions in Ontario, Canada: a one-year study. J Forensic Sci 2015;60:669-74.

27. Government of Canada. Privacy Act. Available at: https://laws-lois. justice.gc.ca/eng/acts/P-21/. Accessed November 30, 2018.

28. Office of the Privacy Commissioner of Canada. Summary of privacy laws in Canada. Available at: https://www.priv.gc.ca/en/privacy-topics/ privacy-laws-in-canada/02_05_d_15/. Accessed November 30, 2018.

29. Accessing Health and Health-Related Data in Canada: the Expert Panel on Timely Access to Health and Social Data for Health Research and Health System Innovation. Ottawa, ON: The Council of Canadian Academies, 2015.

\section{Appendix 1. Canadian Sudden Cardiac Arrest Network (C-SCAN) Investigators}

Brian Grunau, Jim Christenson, Christopher Fordyce, Ian Blanchard, Gerald Lazarenko, Allan DeCaen, Sean Van Diepen, Justin Ezekowitz, Jeff Bakal, Elizabeth Brooks-Lim, Philip Davis, Paul Olszynski, Jodi Garner, Sheldon Cheskes, Dirk Huyer, Kris Cunningham, Laurie Morrison, Katie Dainty, Dennis Ko, Nigel Tan, Patrick R. Lawler, Christian Vaillancourt, Manya Charette, Martin Green, Sharmila Udupa, Eli Segal, Luc Demontigny, Brian Potter, John Sapp, Jan Jensen, Alix Carter, Matthew Bowes, Sean Connors, Stephen Duffett, Brian Metcalfe, Simon Avis, and Aaron Sibley.

\section{Supplementary Material}

To access the supplementary material accompanying this article, visit the online version of the Canadian Journal of Cardiology at www.onlinecjc.ca and at https://doi.org/10. 1016/j.cjco.2019.01.005. 